\title{
ELABORATION OF A CLINICAL-SURGICAL MODEL OF SPARING RADIOTHERAPY OF PECTORAL MUSCLES AND ANTERIOR THORACIC WALL IN PATIENTS SUBMITTED TO SKIN AND NIPPLE PRESERVING MASTECTOMY WITH IMMEDIATE BREAST RECONSTRUCTION
}

Maximiliano Cassilha Kneubil

${ }^{1}$ Hospital Tacchini - Bento Gonçalves (RS), Brazil.

Introduction: The rates of adenomastectomy with immediate breast reconstruction (IBR) in patients with breast cancer (BC), in its early stage, are increasing. Lately, the number of patients who undergo radiotherapy (RT) in this scenario has also increased, since patients submitted to mastectomy who present a compromised axillary lymph node already can have clinical benefits from RT. The routine irradiation of muscles in the anterior thoracic wall generates many complications in patients submitted to mastectomy with IBR. In the literature, the local recurrence rate in the general muscle is of approximately $0.6 \%$. The essential point that comes to light is that the $0.6 \%$ recurrence rate justifies the routine irradiation of the major pectoral muscle in patients who underwent mastectomy with IBR. The selection of initial BC tumors distant from the major pectoral muscle can eventually spare the pectoral muscle in the planning of the target volume in RT, thus considerably reducing the postoperative complication rates, such as capsular contracture. The ESTRO (European Society for Radiotherapy and Oncology) guidelines recommend sparing the major pectoral muscle, considering the anterior surface of the major pectoral muscle as the dorsal edge in the planning of the target volume of the thoracic wall. The recommendation is: unless the invasion has been demonstrated (tumor stage T4a and T4c), there is no reason to include the major pectoral muscle and the ribs in the thoracic wall in the routine, while planning the target volume. In this context, we elaborated a new radiosurgery model called CHRISTMAS (Chest muscle spaRIng post-MAStectomy radiotherapy). Objective: To elaborate a clinical-surgical model of Sparing Radiotherapy of Major Pectoral Muscle and Anterior Thoracic Wall in patients submitted to adenomastectomy with IBR. Methods: We selected a patient with BR, clinical staging T1N1, more than $2 \mathrm{~cm}$ away from the major pectoral muscle, in the Mastology service of Hospital Tacchini. This patient was submitted to adenomastectomy with IBR with expander. Intraoperative radiotherapy with electrons was performed in the nipple-areolar complex, with posterior adjuvant RT of lymph chains through intensity-modulated RT (IMRT), sparing the tissue adjacent to the breast implant (pectoral muscle to nipple level, minor pectoral muscle and anterior thoracic wall). Results: The patient evolved without complications.This new technique has drastically reduced the irradiation rate of the tissue adjacent to the breast implant, besides lungs, pleura and heart. Conclusion: The CHRISTMAS radiosurgery model is technically viable, regarding logistics and radiotherapy planning, and is part of a research project of our institution, in which patients with indication for adenomastectomy, IBR and adjuvant RT with initial BC, distant from the pectoral muscle, will be randomized for conventional RT versus the CHRISTMAS model, comparing the complication and locoregional recurrence rates. 